\title{
Theoretical investigation of fission fragment kinetic energy distributions in the symmetric mass region for ${ }^{233} \mathrm{U}\left(n_{t h}, f\right)$
}

\author{
Abdelaziz Chebboubi $^{1,2, a}$, Olivier Serot ${ }^{1}$, Grégoire Kessedjian ${ }^{2}$, Olivier Litaize $^{1}$, Aurelien Blanc ${ }^{3}$, David Bernard ${ }^{1}$, \\ Herbert Faust $^{3}$, Sylvain Julien-Laferrière ${ }^{1,2}$, Ulli Köster ${ }^{3}$, Alain Letourneau ${ }^{4}$, Thomas Materna ${ }^{4}$, Olivier Méplan ${ }^{2}$, \\ Paolo Mutti ${ }^{3}$, Michal Rapala ${ }^{4}$, and Christophe Sage ${ }^{2}$ \\ ${ }^{1}$ CEA, DEN, DER, SPRC, Cadarache, Physics Studies Laboratory, 13108 Saint-Paul-les-Durance, France \\ 2 LPSC, Université Grenoble-Alpes, CNRS/IN2P3, 38026 Grenoble Cedex, France \\ 3 Institut Laue-Langevin, 38042 Grenoble Cedex 9, France \\ ${ }^{4}$ CEA, DRF, IRFU, SPhN, Saclay, 91191 Gif-sur-Yvette, France
}

\begin{abstract}
Fission yields are essential for nuclear reactor studies (decay heat, fuel inventory...) and constitute also one of the main observables needed to improve our understanding of the fission process. The symmetric mass region is of particular interest due to various intriguing properties of the fission fragments already reported in the literature : inversion of the nuclear charge polarization, large width of the fission fragment kinetic energy distribution, strong change of the prompt neutron multiplicity, etc. Recently, measurements of fission yields and kinetic energy distributions in the symmetric mass region were achieved at the LOHENGRIN mass spectrometer of the Institut Laue-Langevin (ILL). This experimental work is challenging due to the low counting rate and the appearance of contaminant masses, leading to pronounced components in the fission fragment kinetic energy distribution. Despite removing the undesirable contributions, the fission fragment kinetic energy distributions still show two components, indicating that the fission process could be modal. To go further and better characterize these components a comparison between our experimental data and Monte Carlo calculations (FIFRELIN code) simulating the de-excitation of the fission fragments for different fission channels will be presented and discussed.
\end{abstract}

\section{Introduction}

As computing power grows, studies of innovative nuclear reactor concepts more and more rely on complex multiphysics modelling. These simulations require among others accurate knowledge of fission data in the actinide region. In order to improve nuclear data evaluation, two complementary paths are available. One is related to the experimental aspects. The development of new detectors combined with new methodologies aimed to better estimate and control systematic uncertainties. The construction of a covariance matrix associated to the experimental data is the keystone of these efforts. Another way for improving the evaluation of nuclear data is to develop the modelling of the fission phenomenon. The use of more fundamental physics models try to improve the consistency of fission data. Therefore, the uncertainties of these data would be reduced as long as our knowledge of the fission process would get better.

In this way, the symmetric region is a good laboratory to test model hypotheses. Indeed this region shows specific behaviour (inversion of the nuclear charge polarization, large width of the fission fragment kinetic energy distribution, strong change of the prompt neutron multiplicity...).

Recently new measurements on the LOHENGRIN mass spectrometer [1] of the Institut Laue-Langevin (ILL)

\footnotetext{
a e-mail: abdelhazize.chebboubi@cea.fr
}

were achieved. Section 2 presents briefly the experimental apparatus as well as methodology and results. The interpretation of these data was done by combining the fission channels predicted by Brosa [2] with the Monte Carlo code FIFRELIN [3-5]. In order to compare these simulated data with experimental data, energy loss corrections must be applied. All the details are presented in Sect. 3. Section 4 shows the comparison between the simulated and experimental data. Finally the conclusion of this work and its perspectives is exposed in Sect. 5.

\section{Experimental side}

Since 2010 our collaboration has developed a measurement program on fission fragment characteristics (yields, kinetic energy distributions...) at the LOHENGRIN spectrometer of the ILL [6]. The LOHENGRIN fission fragment separator is a double parabola mass spectrometer which combines an electric and a magnetic sectors. Fission fragments, characterized by their mass $A$, nuclear charge $Z$ and kinetic energy $E_{k}$, emerge from the fissile target, placed near the reactor core (under a flux of $\sim 5 \times$ $10^{14} \mathrm{~cm}^{-2} \mathrm{~s}^{-1}$ thermal neutrons), with an ionic charge $q$. The selection of the instrument is achieved following $A / q$ and $E_{k} / q$ ratios.

One of the main observables measured with the LOHENGRIN spectrometer is the mass yields. New methodologies were developed in order to improve the precision reachable with this instrument [7-10]. The 
difference in the descent to the symmetry region seems to be coming from the appearance of a tail at low kinetic energy. It has been shown that this component is the consequence of a change of the fission fragment ionic charge due to their collision with the LOHENGRIN residual gas $[9,11]$. In order to remove this undesired component, an indicator of contamination as well as a procedure to correct the kinetic energy were developed $[9,11]$. Nevertheless this component still persists and simple generalisation tends to confirm the consistency of these structures $[9,11]$.

\section{Simulation side}

The purpose of this work is to combine the Monte Carlo code FIFRELIN (described in the Sect. 3.1) with the Brosa model (see Sect. 3.2 for details) in order to get the kinetic energy distribution per mass. A procedure of energy loss correction was developed to compare the simulated data with the experimental ones (see Sect. 3.3) and conclude on the compatibility between the observed components in the experimental kinetic energy distributions and the Brosa modes.

\subsection{Description of FIFRELIN}

FIFRELIN is a Monte Carlo code developed at CEA Cadarache [3-5]. This code aims to describe the de-excitation path of a fission fragment since its creation until reaching a nuclear state leading to a $\beta$ decay of the atomic nucleus. It is based on the formalism of nuclear realization [12] generalised to neutron $/ \gamma$ coupled emission [5]. Combination of input files and models are used to achieve this purpose.

- The first step is to determine the production rate of each fission fragments $(A, Z)$. Pre-neutron mass yield combined with the Wahl model [13] are used in the present work for the isotopic distribution per mass.

- The second step is to set the excitation and kinetic energy of each fission fragment pair. Pre-neutron kinetic energy distributions (mean and standard deviation) per mass are the starting point. The repartition of the total excitation energy is done by using a temperature ratio law with two free parameters: $\mathrm{RT}_{\min }$ and $\mathrm{RT}_{\max }$. Then, the angular momentum of the fission fragments $(J)$, are sampled according to the following distribution:

$$
P(J) \propto(2 J+1) \exp \left(-\frac{(J+1 / 2)^{2}}{2 \sigma^{2}}\right)
$$

where $\sigma^{2}$ stands for the spin cut-off parameter.

- The third step is to build the nuclear structure scheme for each fission fragment created. Above a cut-off energy ( $E_{\text {cut-off }}^{\mathrm{RIPL}}$ ), the discrete experimental nuclear scheme coming from the RIPL-3 database [14] is completed with discrete levels with a model of level density (here CGCM [15]) up to a second cut-off energy $\left(E_{\text {bin }}\right)$. Above this energy, the nuclear level scheme is described as a continuum.

- The last step is to determine the de-excitation cascade which involves $\gamma$ and neutron emission.
The probability to decay through the emission of a neutron is calculated using neutron transmission coefficients which were determined by using an external code (TALYS/ECIS [16]) and depends on optical model parametrizations. Thanks to the models of $\gamma$ strength function (here EGLO [17]) the probability for an initial nuclear state to decay to a final step by emitting a $\gamma$-ray is calculated.

In this work, only the post-neutron emission kinetic energy distributions are considered in order to compare FIFRELIN results with experimental kinetic energy distributions.

\subsection{Brosa fission channels}

In their work [2] Brosa et al. state that the mass yields and kinetic energy distributions are ruled by multiple modes and random neck rupture at scission. In this work, we choosed to use the Brosa mode parameters as input data for FIFRELIN. In the case of ${ }^{233} \mathrm{U}$, three fission channels, corresponding to three different scission configurations are used to interpret our experimental results. The superlong (SL) refers to an elongated compound nucleus, whereas Standard modes (StI and StII) are related to more compact configurations. The model predicts the mean value and width of the mass and kinetic energy distributions (for each fission channel) but not the relative weight. The production rate of a fission fragment given its mass $(A)$ and the total kinetic energy (TKE) can be written as follows:

$$
\begin{aligned}
Y(A, \mathrm{TKE})= & \sum_{i=1}^{3} \frac{W_{i}}{\sqrt{2 \pi} \sigma_{A_{i}}} \exp \left(-\frac{\left(A-\overline{A_{i}}\right)^{2}}{2 \sigma_{A_{i}}^{2}}\right) \\
& \times \frac{F(A)}{C_{i} \sqrt{2 \pi} \sigma_{d_{i}} \mathrm{TKE}^{2}} \exp \left(-\frac{\left(\frac{F(A)}{\mathrm{TKE}}-d_{i}\right)^{2}}{2 \sigma_{d_{i}}^{2}}\right) \\
\mathrm{TKE}= & \frac{F(A)}{d}=\frac{A}{A_{C N}} \times \frac{Z_{C N}^{2}\left(1-\frac{A}{A_{C N}}\right) e^{2}}{4 \pi \epsilon_{0} d} \\
C_{i}= & \int_{0}^{\infty} \frac{F(A)}{\sqrt{2 \pi} \sigma_{d_{i}} \mathrm{TKE}^{2}} \exp \left(-\frac{\left(\frac{F(A)}{\mathrm{TKE}}-d_{i}\right)^{2}}{2 \sigma_{d_{i}}^{2}}\right) \mathrm{dTKE}
\end{aligned}
$$

with $d$ the average distance at scission between the charge center of the nascent fragments and $\sigma_{d}$ its width. The parameters $A_{C N}$ and $Z_{C N}$ refer to the compound nucleus mass and nuclear charge respectively.

We remind that FIFRELIN calculations require input data such as pre-neutron emission mass yield and the total kinetic energy mean and width per mass. In the late 60s, Pleasonton [18] measured both quantities for ${ }^{233} \mathrm{U}\left(n_{t h}, f\right)$ reaction. Here, we used the same procedure described by Knitter et al. [19] on ${ }^{235} \mathrm{U}\left(n_{t h}, f\right)$ to extract the experimental characteristics of the fission modes in the case of ${ }^{233} \mathrm{U}\left(n_{t h}, f\right)$ reaction. These data are compiled in Table 1 and compared with theory in the case of ${ }^{235} \mathrm{U}\left(n_{t h}, f\right)$. A more coherent and precise determination of the fission mode characteristics would require a fit of the $Y(A, \mathrm{TKE})$ experimental data which are not available in [18]. Nevertheless, these fitting parameters seem in agreement with the expected value and also with the ones extracted from experimental data in the case of ${ }^{235} \mathrm{U}\left(n_{t h}, f\right)$ [19]. In this work, for each Brosa modes a 
Table 1. Synthesis of the characteristics of the three fission channels extracted from Pleasonton data of $\left({ }^{233} \mathrm{U}\left(n_{\mathrm{th}}, f\right)\right)$, from theoretical consideration and from experimental study of ${ }^{235} \mathrm{U}\left(n_{\text {th }}, f\right)$.

\begin{tabular}{cccccc}
\hline \hline Modes & $W(\%)$ & $\bar{A}(\mathrm{u})$ & $\sigma_{A}(\mathrm{u})$ & $d(\mathrm{fm})$ & $\sigma_{d}(\mathrm{fm})$ \\
\hline \multicolumn{5}{c}{ Extracted from Pleasonton data $\left({ }^{233} \mathrm{U}\left(n_{t h}, f\right)\right)$} & {$[18]$} \\
\hline \hline SL & $0.53 \pm 0.13$ & 117 & $5.4 \pm 1.6$ & $19.3 \pm 0.5$ & $2.0 \pm 0.4$ \\
StI & $24.3 \pm 0.5$ & 133.1 & $2.80 \pm 0.02$ & $16.9 \pm 0.3$ & $1.05 \pm 0.15$ \\
StII & $74.9 \pm 0.5$ & 140.6 & $4.18 \pm 0.02$ & $17.6 \pm 0.4$ & $0.8 \pm 0.3$ \\
\hline \multicolumn{6}{c}{ Theory $\left({ }^{235} \mathrm{U}\left(n_{t h}, f\right)\right)[2,20]$} \\
\hline \hline SL & tbd & 118 & 12.9 & 19.5 & 0.99 \\
StI & tbd & 135 & 3.4 & 16.4 & 0.69 \\
StII & tbd & 141 & 5.9 & 17.6 & 0.83 \\
\hline \multicolumn{6}{c}{} \\
\hline \hline SL & $0.07 \pm 0.01$ & 118 & $4.1 \pm 2.4$ & $19.4 \pm 0.9$ & $1.00 \pm 0.68$ \\
StI & $18.3 \pm 0.3$ & 133.9 & $2.62 \pm 0.63$ & $16.0 \pm 0.1$ & 0.80 \\
StII & $81.4 \pm 0.4$ & 141.1 & $4.96 \pm 0.63$ & $17.5 \pm 0.1$ & 0.69 \\
\hline \hline
\end{tabular}

FIFRELIN calculation is performed. Therefore for each mode, the pre-neutron emission mass yield and the total kinetic energy mean and width per mass are derived from the fitting parameters and used as input data for FIFRELIN

\subsection{From FIFRELIN to LOHENGRIN}

In order to compare the simulated post-neutron emission kinetic energy distributions with experimental distributions, the energy loss through the fissile deposit and its cover must be taken into account. In the 2015 experiment, the ${ }^{233} \mathrm{U}$ target $\left(113 \mu \mathrm{g} \mathrm{cm}{ }^{-2} \leftrightarrow 0.1 \mu \mathrm{m}\right)$ was covered with a thin Ta layer $\left(120 \mu \mathrm{g} \mathrm{cm}^{-2} \leftrightarrow 0.07 \mu \mathrm{m}\right)$. Usually a $0.5 \mu \mathrm{m} \mathrm{Ni}$ foil is used. Here a Ta thin deposit was used in order to reduce the target self sputtering [21] and the energy loss. It turns out that the integrity of the target was preserved and improved its lifetime, whereas the energy loss was reduced by a factor of two on average compared to Ni foils.

Nevertheless, since no precise data exist with Ta, all the calculations were made considering that the experimental \{target-cover set is associated to a single Ni foil, with an effective thickness. Then, a Monte Carlo calculation was developed to correct the simulated data from the energy loss. According to [22] the mean energy loss of a fission fragment through thin foil is:

$$
\begin{gathered}
\frac{\overline{d E}}{d x}=3.07 \times 10^{-4} \times \frac{Z_{N i} Z_{e f f}^{2}}{A_{N i} \beta^{2}} L\left(\mathrm{MeV} \mathrm{cm}^{2} \mathrm{mg}^{-1}\right) \\
Z_{e f f}=Z\left(1-\exp \left(-\frac{v}{v_{0} Z^{\lambda}}\right)\right) \\
L=f(\xi)+\log (\xi)=\sum_{i} a_{i} \log ^{i}(\xi)+\log (\xi) \\
\xi=7.866 \times 10^{7} \times \frac{\beta^{3}}{Z \times I}
\end{gathered}
$$

with $Z_{\text {eff }}$ the average equilibrium charge state, $L$ the stopping number, $I$ the mean excitation potential of the target, $\beta=\frac{v}{c}$ the reduced fission fragment velocity and $v_{0}$ the Bohr velocity. The parameters $\left\{a_{i}, \lambda\right\}$ are obtained by the authors [22] by fitting the energy loss through a thin foil as function of the initial kinetic energy for different ions. However this mean energy loss correction is not enough to

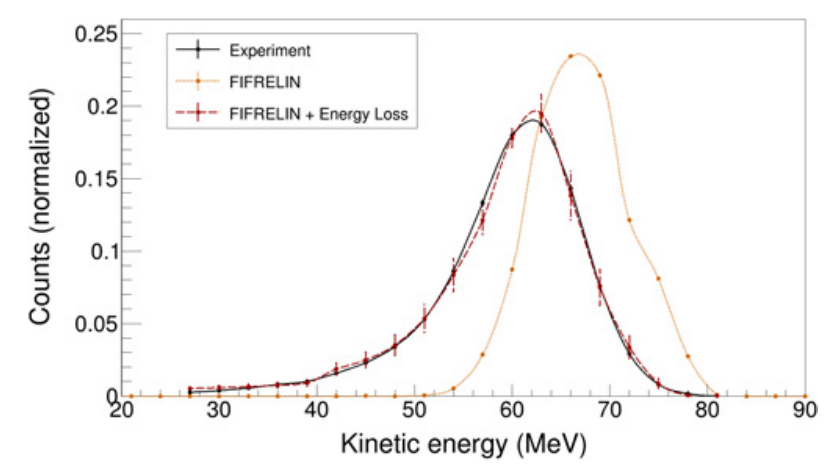

Figure 1. Correction of simulated data with energy loss calculation permits to reproduce, within statistical accuracy, the experimental data for mass $A=136$.

reproduce the experimental data, especially the long tail at low kinetic energy. It has been shown that the energy loss distribution of a high velocity charged particle through a thin foil follows a Landau law [23]:

$$
P\left(\frac{\overline{d E}}{d x}\right)=P(x)=\frac{1}{\pi} \int_{0}^{\infty} e^{-t \log (t)-x t} \sin (\pi t) d t
$$

The sampling over this distribution required two free parameters related to the mean thickness $\bar{e}$ of the target and a scale parameter $\alpha$ related to the distribution width. Finally the corrected energy is:

$$
E_{\text {corrected }}=E_{\text {simulated }}-\rho \bar{e} \frac{d E}{d x}(\bar{e}, \alpha)
$$

with $\rho$ the target density. The free effective parameters $\bar{e}=0.275 \pm 0.030 \mu \mathrm{m}$ and $\alpha=57000 \pm 5000 \mathrm{MeV} \mathrm{cm}^{-1}$ were set in order to reproduce the experimental kinetic energy of the mass $A=136$ [see Fig. 1]. The order of magnitude of these parameters are in complete agreement with the experimental value. These value are now fixed for the other masses.

\section{Comparison with experimental data}

For the region mass around $A=135$, which involves the two standard fission channels, the agreement is quite good. The slight differences can be explained by a time evolution of the effective thickness $\bar{e}$ of the target. Indeed, the target inside the reactor of the ILL is under a hostile environment. For instance the mean measured kinetic energy for the same mass over the whole experiment can be shifted by $2 \mathrm{MeV}$.

For the descent to the symmetry region $(A \sim 125)$, which involves the three fission channels, the agreement is also satisfactory [see Fig. 2]. As expressed before, the parameter $\bar{e}$ is tricky to estimate. A time dependance of this parameter should lead to a better agreement with experimental data, and it corresponds to a perspective of this work.

Finally, the symmetry region $(A \sim 117)$ which should mainly involved superlong fission channel, is in disagreement with experimental data. Different explanations can be proposed. The first one is related to an underestimation of the initial kinetic energy of the SL mode. Moreover, this work is focusing on the post-neutron emission kinetic energy distributions. These distributions are sensitive to 


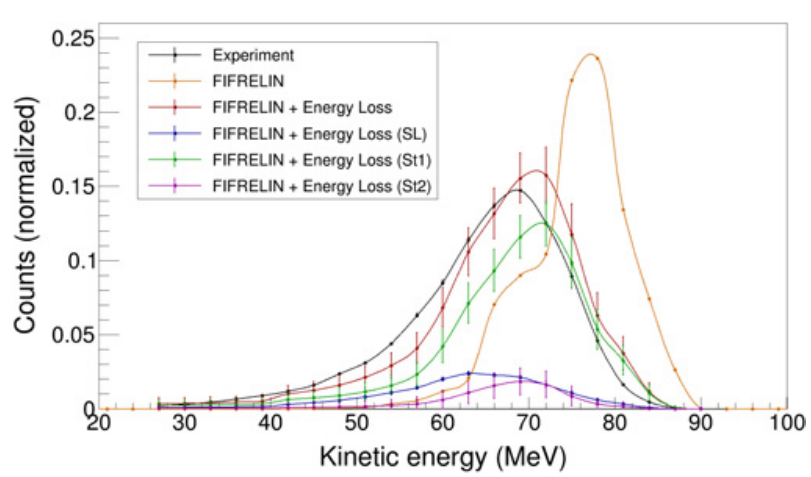

Figure 2. Comparison of the simulated data and experimental data for $A=125$. The contribution of each fission channels after energy loss correction are also shown. A good agreement is found for the descent to symmetry region.

the neutron emission process. In this region, the neutron emission is perhaps underestimated. The weight of the standard fission channels may also be underestimated.

\section{Conclusion and perspectives}

This work presents the path from Brosa fission channels to experimental kinetic energy distribution through the use of the Monte Carlo code FIFRELIN. It shows that both standard modes (StI and StII) combined with an energy loss calculation reproduce with a good agreement the experimental data points. The characteristics of the superlong mode (SL) are still under consideration as they fail to reproduce the data in symmetric region.

Different ways of improvement were highlighted. Taking into account the time dependency of the target thickness through the parameter $\bar{e}$ should improve the agreement between simulated and experimental data. Using the experimental kinetic energy data to determine the characteristics of Brosa modes and look at the consequences on other observables calculated with FIFRELIN (yields, sawtooth, ... ) may lead to a new way to interpret experimental data. Finally the FIFRELIN free parameters (see Sect. 3.1) can also be specific for each Brosa fission channels [24].
This work was supported by CEA, the University of Grenoble Alpes, IN2P3, "le défi NEEDS". The authors are grateful for the support of the ILL and all the staff involved from CEA-Cadarache and LPSC.

\section{References}

[1] P. Armbruster et al., Nucl. Instrum. Meth. 139, 213 (1976)

[2] U. Brosa et al., Phys. Rep. 197(4), 167 (1990)

[3] O. Litaize, O. Serot, Phys. Rev. C 82, 054616 (2010)

[4] O. Litaize et al., EPJ A 51(12), 1 (2015)

[5] D. Regnier et al., Comput. Phys. Commun. 201, 19 (2016)

[6] O. Serot et al., Nucl. Data Sheets 119, 320 (2014)

[7] F. Martin, Phd thesis, Université Grenoble Alpes (2013)

[8] F. Martin et al., Nucl. Data Sheets 119, 328 (2014)

[9] A. Chebboubi, Phd thesis, Université Grenoble Alpes (2015)

[10] G. Kessedjian et al., This conf. (2016)

[11] A. Chebboubi et al., EPJ Web Conf. 111, 08002 (2016)

[12] F. Bečvář, Nucl. Instrum. Meth. A 417, 434 (1998)

[13] A.C. Wahl, At. Data. Nucl. Data Tables 39(1), 1 (1988)

[14] R. Capote et al., Nucl. Data Sheets 110(12), 3107 (2009)

[15] A. Gilbert, A.G.W. Cameron, Can. J. of Phys. 43(8), 1446 (1965)

[16] A. Koning et al., TALYS-1.0 (EDP Sciences, 2007), pp. 211-214

[17] J. Kopecky, M. Uhl, Phys. Rev. C 41, 1941 (1990)

[18] F. Pleasonton, Phys. Rev. 174, 1500 (1968)

[19] H.H. Knitter et al., Z. Naturforsch. A 42(8), 786 (1987)

[20] S. Grossmann et al., Nucl. Phys. A 481(2), 340 (1988)

[21] U. Köster et al., Nucl. Instrum. Meth. A 613(3), 363 (2010)

[22] G. Knyazheva et al., Nucl. Instrum. Meth. B 248(1), 7 (2006)

[23] L. Landau, J. Phys.(USSR) 8, 201 (1944)

[24] O. Serot et al., Phys. Procedia 59, 132 (2014) 